\title{
Efeito da adubação nitrogenada na produção de amaranto
}

\section{Influence of nitrogen fertilization on yield of amaranth}

\author{
Telma Regina Brambilla ${ }^{1}$; Ana Paula Brambilla Constantino ${ }^{2}$; \\ Pérsio Sandir D'Oliveira ${ }^{3^{*}}$
}

\begin{abstract}
Resumo
Este trabalho foi realizado para avaliar o efeito da adubação nitrogenada em cobertura, na produção de amaranto em Maringá, norte do Paraná. Foi realizado em condições de campo, no delineamento inteiramente casualizado, com quatro tratamentos e três repetições por tratamento. As sementes foram semeadas em linhas espaçadas a cada $0,5 \mathrm{~m}$. A adubação de plantio foi feita com NPK 10-10-10 (350 $\left.\mathrm{kg} \mathrm{ha}^{-1}\right)$. Aos 15 dias após a emergência, foi feito o desbaste para deixar uma população de 20 plantas $\mathrm{m}^{-1}$. Aos 30 dias após a emergência, foi aplicada adubação nitrogenada de cobertura (uréia, $45 \% \mathrm{~N}$ ), nas doses $0 ; 50 ; 100 ; 200 \mathrm{~kg} \mathrm{~N} \mathrm{ha}^{-1}$. Aos 114 dias após a emergência, foram medidos os parâmetros: altura da planta; diâmetro do colmo; produção de biomassa fresca e seca da parte aérea; e produção de grãos. A altura da planta e a produção de grãos apresentaram uma resposta quadrática. Não houve diferenças significativas para o diâmetro do colmo. A biomassa da parte aérea (fresca e seca) teve uma resposta linear. $\mathrm{O}$ amaranto respondeu favoravelmente à adubação nitrogenada de cobertura.
\end{abstract}

Palavras-chave: Amaranthus, nutrição mineral, produção vegetal

\begin{abstract}
This work was carried out to study the effect of nitrogen fertilization on grain amaranth yield, in Maringá, north of Paraná state. It was done in field conditions, using a complete randomized design, with four treatments and three replicates. Seeds were sowed in lines, spaced each $0.5 \mathrm{~m}$. Fertilization was done with NPK 10-10-10 (350 kg/ha). Fifteen days after germination there was left a population of 20 plants $\mathrm{m}^{-1}$. Thirty days after germination fields received nitrogen fertilizer (urea, $45 \% \mathrm{~N}$ ), and doses were 0 ; $50 ; 100 ; 200 \mathrm{~kg} \mathrm{~N} \mathrm{ha}^{-1}$. Plants were cut 114 days after germination, to measure the following parameters: plant height; stem diameter; fresh and dry biomass yield of stems and leaves; and seed yield. Plant height and grain production showed quadratic response. Stem diameter showed no differences. Biomass production (fresh and dry) showed linear response. Yield of amaranth grains answered positively to nitrogen fertilization.
\end{abstract}

Key words: Amaranthus, mineral nutrition, crop yield

1 Produtora Rural e Acadêmica do Curso de Tecnologia em Gestão de Agronegócio no CESUMAR, Maringá. E-mail: telmabrambilla@hotmail.com.

2 Acadêmica do Curso de Agronomia da Universidade Estadual de Maringá. E-mail: anapaulabrambilla@hotmail.com.

${ }^{3}$ Engenheiro Agrônomo, Doutor, Professor e Orientador do presente trabalho. CESUMAR, Maringá. E-mail: psandir@cesumar.br.

* Autor para correspondência 


\section{Introdução}

O amaranto (família Amaranthaceae, gênero Amaranthus sp.) é uma planta dicotiledônea, de porte herbáceo, com inflorescência tipo capítulo, cacho ou panícula, com folhas inteiras, de disposição espiralada ou oposta cruzada, sem estípulas (JOLY, 2002; AMAYA-FARFAN; MARCÍLIO; SPEHAR, 2005; COSTA; BORGES, 2005). Tanto as folhas como as sementes podem ser usadas como alimento, e as espécies mais cultivadas do gênero são: $A$. cruentus (México, África, Caribe, Ásia e América do Sul), A. caudatus (América do Sul), A. hypochondriacus (Índia, México e Estados Unidos) e A. tricolor (China, Índia e Sudoeste Asiático) (KAUFFMAN; WEBER, 1990; MYERS, 1996, 1998; SPEHAR; CABEZAS, 2001; GUADARRAMA; SALGADO; SEDEÑO, 2002; CHAKHATRAKAN， 2003; AMAYA-FARFAN; MARCÍLIO; SPEHAR, 2005; COSTA; BORGES, 2005; DOMINGOS et al., 2005).

$\mathrm{Na}$ América Latina, o cultivo do amaranto data de muito antes da chegada dos conquistadores espanhóis, com seu apogeu durante as civilizações asteca, inca e maia, onde era considerado como alimento sagrado: consumia-se o grão de amaranto, moído e misturado com o sangue de sacrifícios humanos, em cerimônias religiosas; esta prática foi banida pelos espanhóis, e o cultivo desta planta praticamente desapareceu (MYERS, 1996; AMAYA-FARFAN; MARCÍLIO; SPEHAR, 2005; COSTA; BORGES, 2005).

Por volta dos anos 1960, o amaranto foi levado para a Europa e, por volta de 1980, alcançou as montanhas do Nepal e parte do Leste da África, sendo cultivado na China, África, Europa e na América do Norte (MYERS, 1996; COSTA; BORGES, 2005). Atualmente, os Estados Unidos lideram a produção mundial de amaranto, utilizando o grão como alimento em vários produtos; porém, na última década, a China se tornou o maior produtor mundial de amaranto, que é usado como forragem para o gado e na alimentação de suínos. Na Índia, os grãos de amaranto são usados como farinha, para alimentar a população mais pobre (MYERS, 1996; AMAYA-FARFAN; MARCÍLIO; SPEHAR, 2005; COSTA; BORGES, 2005).

$\mathrm{O}$ interesse crescente no amaranto é justificado pelo alto valor protéico (14 a 16\%) do grão, de alta qualidade biológica devido ao seu conteúdo de lisina e outras aminoácidos essenciais, além de fornecer fibras e minerais, principalmente cálcio (MYERS, 1996; ERASMO et al., 2004; AMAYA-FARFAN; MARCÍLIO; SPEHAR, 2005; COSTA; BORGES, 2005; DOMINGOS et al., 2005). Com a maior procura por dietas alimentares mais saudáveis, especialmente pelas pessoas com intolerância ao glúten, o grão de amaranto pode ser usado na fabricação de farinhas, cereais matinais, massas e biscoitos (AMAYA-FARFAN; MARCÍLIO; SPEHAR, 2005; COSTA; BORGES, 2005).

$\mathrm{O}$ amaranto apresenta facilidade de crescimento em altas temperaturas e baixa precipitação, e existem variedades tolerantes a solos salinos e/ou com presença de alumínio tóxico (ERASMO et al., 2004); além da formação de palha, a planta também tem potencial forrageiro (BRENNER; WILLIAMS, 1995; SPEHAR et al., 2003). No Brasil, a Embrapa Cerrados (DF) testou várias espécies de amaranto, para produção de alimentos, diversificação de grãos e adubação verde na entressafra, além de uso no plantio direto (SPEHAR; SANTOS; SOUZA, 1997; SPEHAR; CABEZAS, 2001; SPEHAR; TEIXEIRA, 2002). O cultivar BRS Alegria já está disponível (SPEHAR et al., 2003).

O nitrogênio é um macronutriente, essencial para as plantas e, exceto as leguminosas, que conseguem obter o nitrogênio de que necessitam através da simbiose, as demais precisam receber este elemento, seja por adubação orgânica, seja por adubação mineral (TAIZ; ZEIGER, 2004). Como qualquer outra planta cultivada, o amaranto responde à fertilidade do solo, e o nitrogênio provou ser o principal fator limitante (WEBER, 1989 apud MYERS, 1998). 
Existem poucos trabalhos sobre adubação do amaranto, mas os resultados encontrados pela pesquisa reforçam a importância do nitrogênio para esta planta: nos Estados Unidos, verificou-se que a adubação nitrogenada deve estar entre 112

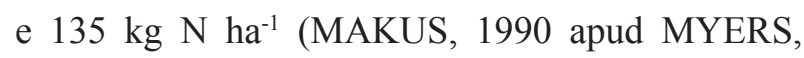
1998; PUTNAM, 1990 apud DOMINGOS et al., 2005; SCHULZ-SCHAEFFER et al., 1989). Na Tailândia, Amaranthus tricolor, cultivado para o consumo das folhas, recebeu doses de crescentes de $\mathrm{N}\left(0 ; 15 ; 187,5 ; 250\right.$; e $\left.312,5 \mathrm{~kg} \mathrm{~N} \mathrm{ha}^{-1}\right)$, e verificouse aumento linear da altura, diâmetro do colmo, número de folhas por planta e biomassa fresca (CHAKHATRAKAN, 2003).

No Brasil, ensaio para avaliar a produção de variedades de Amaranthus spp. no sul do Tocantins empregou adubação de plantio com NPK (05-2515), na dose de $300 \mathrm{~kg} \mathrm{ha}^{-1}$, e adubação de cobertura aos 30 dias após a emergência, com uréia $(45 \% \mathrm{~N})$, na dose de $45 \mathrm{~kg} \mathrm{ha}^{-1}$ (ERASMO et al., 2004).

Em geral, experimentos com amaranto, realizados nos trópicos, mostram respostas inconsistentes à adubação nitrogenada, refletindo a diversidade de ambientes (clima e solo) onde os experimentos foram realizados. Além disso, a ocorrência de tombamento no amaranto, pode ser um problema; daí a importância de determinar se o tombamento aumenta com as doses de nitrogênio (MYERS, 1998).

Existe uma relação entre o teor de matéria orgânica do solo e a necessidade de adubação nitrogenada para a cultura do amaranto: solo com baixos teores de matéria orgânica requer doses mais elevadas de fertilizantes nitrogenados, tanto no plantio quanto em cobertura (CHAKHATRAKAN, 2003).

O objetivo deste trabalho foi avaliar o efeito da adubação nitrogenada de cobertura na produção de amaranto, cultivado no município de Maringá, norte do Paraná.

\section{Material e Métodos}

$\mathrm{O}$ experimento foi realizado em canteiros experimentais no campus-sede do CESUMAR, em Maringá. A cidade está localizada a 554 m acima do nível do mar, e suas coordenadas geográficas são $23^{\circ}$ $24^{\prime} 43^{\prime \prime}$ S e $51^{\circ} 55^{\prime} \mathrm{W}$. O clima, pela classificação de Köeppen (AYOADE, 2003), é do tipo Cfa(h): clima subtropical mesotérmico, com verões chuvosos e geadas pouco freqüentes. Antes do plantio, foram coletadas amostras de solo, para análise de fertilidade, e os resultados foram: $\mathrm{pH}$ (água): 6,2; $\mathrm{H}+\mathrm{Al}:$ 30,6 $\mathrm{mmol}_{\mathrm{c}} \mathrm{dm}^{-3}$; matéria orgânica: 9,48 g $\mathrm{dm}^{-3}$; P: 28,66 mg dm $\mathrm{m}^{-3}$; K: 3,9 mmol $\mathrm{dm}^{-3}$; Ca: 54,8 $\mathrm{mmol}_{\mathrm{c}} \mathrm{dm}^{-3}$; $\mathrm{Mg}: 13,7 \mathrm{mmol}_{\mathrm{c}} \mathrm{dm}^{-3}$; micronutrientes (mg dm${ }^{-3}$ ): $\mathrm{Cu}$ 8,90; Fe 53,00; Mn 61,00; Zn: 4,70. $\mathrm{O}$ teor de $\mathrm{Al}^{3+}$ foi zero.

Sementes de amaranto BRS Alegria foram fornecidas pela EMBRAPA Cerrados. Foi usado o espaçamento de $0,5 \mathrm{~m}$ entre linhas, com stand final de 20 plantas $\mathrm{m}^{-1}$. O experimento seguiu um delineamento inteiramente casualizado, com três repetições por tratamento (GOMES, 1985). A adubação de plantio foi feita com NPK (10-10-10), na dose de $350 \mathrm{~kg} \mathrm{ha}^{-1}$. A semeadura foi realizada em 31/10/2006. O desbaste foi realizado aos quinze dias após a emergência, para se obter uma população de 20 plantas $\mathrm{m}^{-1}$. Aos 30 dias após a emergência, foi aplicada adubação nitrogenada de cobertura (uréia, $45 \% \mathrm{~N}$ ), nas doses: 0; 50; 100; e $200 \mathrm{~kg}$ de $\mathrm{N} \mathrm{ha}^{-1}$, sem incorporação do fertilizante.

Os canteiros, com área de $20 \mathrm{~m}^{2}$, foram monitorados durante todo o período, para observar a ocorrência de pragas e doenças, e replantio das plântulas. Capinas manuais foram realizadas semanalmente, até os 30 dias após a emergência. A irrigação foi feita com regador, duas vezes por dia, até os 30 dias após a emergência. Não houve necessidade de controle químico de pragas e doenças.

Aos 114 dias após a emergência, foram medidos os parâmetros: altura da planta; diâmetro do colmo; 
produção de biomassa da parte aérea fresca e seca; e produção de grãos. Foram desprezadas as plantas da primeira e última fileiras, para evitar o efeito bordadura. A altura foi medida com trena metálica, graduada em $\mathrm{cm}$. O diâmetro do colmo foi medido com paquímetro, a $5 \mathrm{~cm}$ do solo. Após a medição da altura, todas as plantas foram cortadas com tesoura de poda, a $5 \mathrm{~cm}$ do solo, e foram pesadas, separadamente, a parte aérea (folha + colmo) e a panícula. As pesagens foram feitas em balanças semi-analíticas, para determinar a biomassa fresca e a produção de grãos. Alíquotas da biomassa fresca de cada canteiro, pesando $100 \mathrm{~g}$, foram adicionadas em sacos de papel, previamente pesados, e levadas a estufas de ventilação forçada a $45^{\circ} \mathrm{C}$ até peso constante; o material foi novamente pesado e, por diferença entre o peso inicial e o peso final, foi calculado o teor de matéria seca. Este valor foi multiplicado pela biomassa fresca, para se obter a produção de biomassa seca.

Os dados obtidos foram submetidos à análise de variância pelo programa de computador SAEG e, a partir dos resultados da análise estatística, foram construídas as equações de regressão linear.

\section{Resultados e Discussão}

A altura da planta $(\mathrm{m})$ respondeu à adubação nitrogenada, e foi de 2,1 $\mathrm{m}$ (testemunha) até 2,5 m (100 kg ha-1) aos 114 dias após a emergência; a dose maior (200 $\left.\mathrm{kg} \mathrm{ha}^{-1}\right)$ não resultou em maior altura (Figura 1). Resultados semelhantes foram encontrados no Tocantins, onde adubação com NPK (05-25-15), na dose de $400 \mathrm{~kg} \mathrm{ha}^{-1}$ produziu plantas com maior altura média (DOMINGOS et al., 2005). No México, aplicação de $250 \mathrm{~kg} \mathrm{~N}^{-1}$ resultou em plantas com altura de 2,0 m (GUADARRAMA; SALGADO; SEDEÑO, 2002). Além do efeito direto da adubação, pode haver influência dos fatores ambientais: Amaranthus hypocondriacus L. cultivado no Quênia, África, produziu plantas baixas ( $<1,0 \mathrm{~m})$; quando o mesmo foi cultivado na Pensilvânia, EUA, produziu plantas altas $(>2,0 \mathrm{~m})$ (KAUFFMAN; WEBER, 1990).

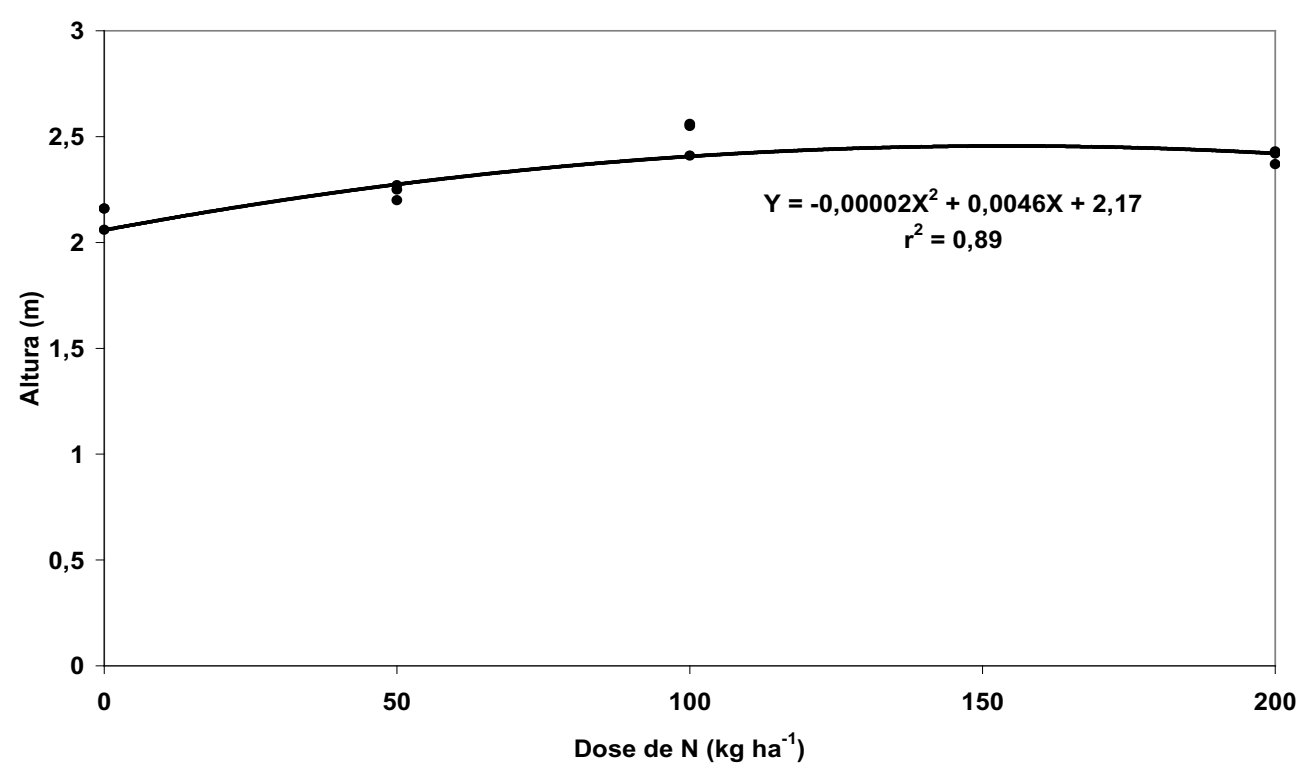

Figura 1. Altura de plantas de amaranto em função da dose de $\mathrm{N}\left(\mathrm{kg} \mathrm{ha}^{-1}\right)$ em cobertura. 
A biomassa da planta também respondeu à adubação nitrogenada, e foi verificado um efeito linear (Figura 2): quanto maior a dose de $\mathrm{N}$, maior a produção de biomassa, que foi de $40,4 \mathrm{t} \mathrm{ha}^{-1}$ (testemunha) até 47 tha $^{-1}\left(200 \mathrm{~kg} \mathrm{Nha}^{-1}\right)$. Trabalhando com adubação com NPK (05-25-15), Domingos et al. (2005) verificaram aumento de produção de biomassa fresca e seca de plantas individuais com doses crescentes de adubo, e o melhor resultado foi observado na cultivar BRS Alegria. Esta cultivar alcançou uma produção de biomassa seca total de 5,65 tha-1 (SPEHAR et al., 2003).

A produção de matéria seca do amaranto chega a ser maior que a produção do milheto, sorgo ou milho (TEIXEIRA; SPEHAR; SOUZA, 2003). A cultivar de amaranto Oscar Blanco apresentou maior produção de biomassa seca aos 55 dias após a emergência (ERASMO et al., 2004).

Esta produção de biomassa sugere o cultivo do amaranto no norte do Paraná, em programas de plantio direto, pois, nesta prática, é necessário empregar culturas que produzam grande quantidade de palha (SPEHAR; SANTOS; SOUZA, 1997). Assim, o amaranto pode substituir culturas como aveia, milheto e outras usadas rotineiramente nesta prática conservacionista, consagrada entre os produtores paranaenses.

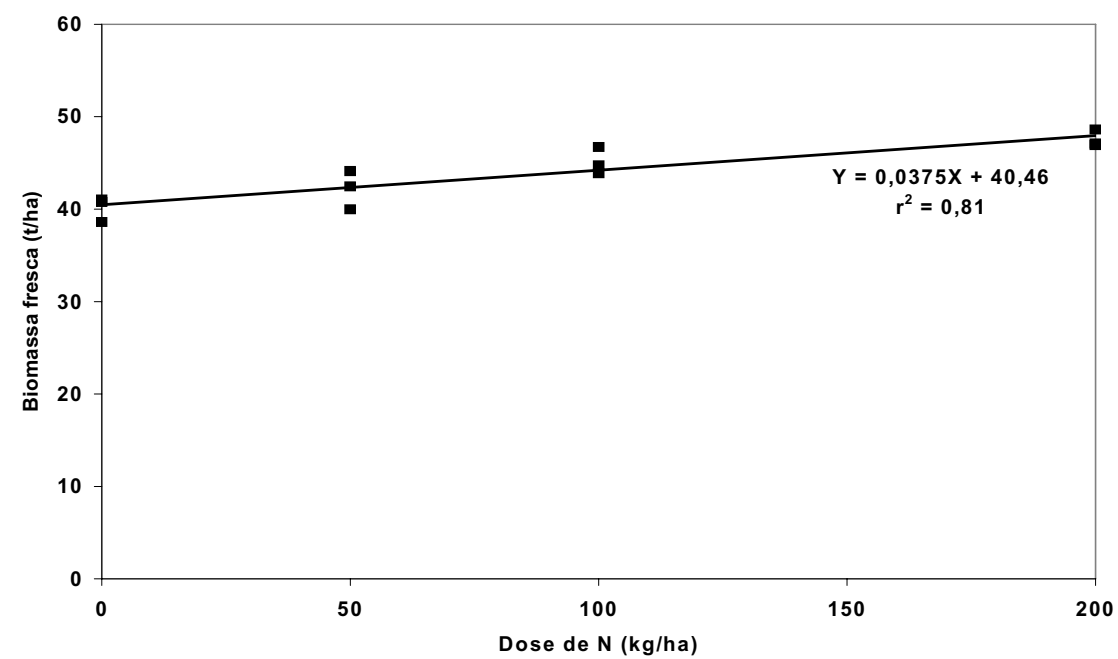

Figura 2. Produção de biomassa fresca em função da dose de $\mathrm{N}\left(\mathrm{kg} \mathrm{ha}^{-1}\right)$ em cobertura.

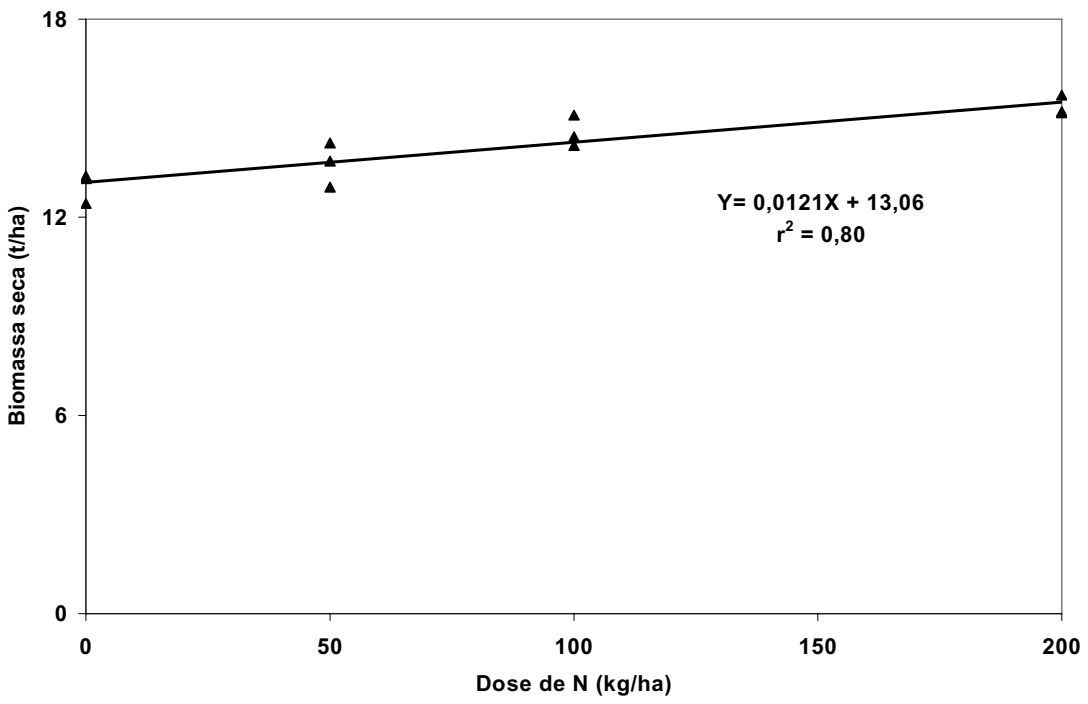

Figura 3. Produção de biomassa seca em função da dose de $\mathrm{N}\left(\mathrm{kg} \mathrm{ha}^{-1}\right)$ em cobertura. 


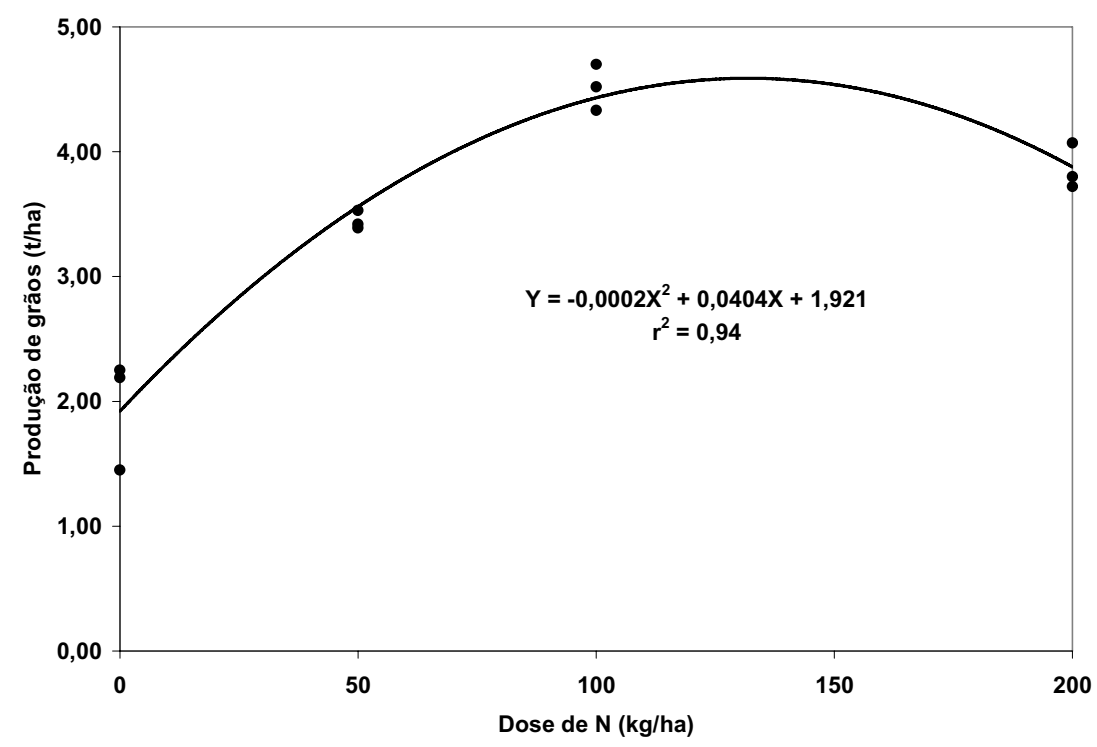

Figura 4. Produção de grãos em função de dose de $\mathrm{N}\left(\mathrm{kg} \mathrm{ha}^{-1}\right)$ em cobertura.

A produção de grãos apresentou uma resposta quadrática, com aumento até $100 \mathrm{~kg} \mathrm{~N} \mathrm{ha}^{-1}$ (Figura 4). Os resultados são semelhantes aos encontrados por Myers (1998), que testou três cultivares de amaranto e cinco níveis de adubação nitrogenada, e observou aumento até a dose de $90 \mathrm{~kg} \mathrm{~N} \mathrm{ha}^{-1}$, mas não acima desta. Os resultados são superiores aos encontrados por Teixeira, Spehar eSouza (2003), em solo de Cerrado.

Por outro lado, no Arkansas (EUA), foram cultivados A. cruentus e A. hypocondriacus em três doses de $\mathrm{N}\left(0 ; 100\right.$ e $\left.200 \mathrm{~kg} \mathrm{ha}^{-1}\right)$, e embora a produtividade no $1^{\circ}$ ano tenha dobrado com a dose de $100 \mathrm{~kg} \mathrm{ha}^{-1}$, no $2^{\circ}$ ano não houve resposta (ENDRES, 1986 apud KAUFFMANN; WEBER, 1990). Foi registrado efeito linear na produção de grãos de amaranto, até a dose de $500 \mathrm{~kg}$ de $\mathrm{N} \mathrm{ha}^{-1}$ (ERASMO et al., 2004). Na Bolívia, o mesmo efeito foi observado com adubação orgânica (esterco ovino) ou mineral NPK (40-40-20 ou 80-80-40) (APAZA-GUTIERREZ et al., 2002).

Com relação ao diâmetro do colmo, não foram observadas diferenças entre os tratamentos, e este parâmetro ficou, na média, em $2,8 \mathrm{~cm}$. Este índice é útil para estimativa de tombamento das plantas. No presente ensaio, não foi registrada ocorrência de tombamento; é provável que, no amaranto, o espaçamento entre fileiras e a densidade de plantas sejam mais importantes no diâmetro do colmo do que a adubação mineral (HENDERSON; JOHNSON; SCHNEITER, 2000; APAZA-GUTIERREZ et al., 2002).

Deve-se levar em conta a alta fertilidade do solo do local, bem como condições climáticas favoráveis; ao passo que os experimentos com amaranto no Cerrado enfrentam condições adversas: baixa fertilidade e toxicidade de alumínio. Além disso, o cultivo ocorreu em condições de disponibilidade de água, ao passo que, no Cerrado, existe uma estação seca bem definida. As condições do presente experimento foram extremamente favoráveis para o cultivo da planta.

\section{Conclusões}

$\mathrm{O}$ amaranto respondeu positivamente à adubação nitrogenada de cobertura, e para a produção de grãos, não é preciso aplicar doses acima de 100 $\mathrm{kg} \mathrm{ha}^{-1}$. Se o objetivo for a produção de biomassa, como produção de forragem e palha para plantio direto, podem ser usadas doses maiores. A planta pode ser mais uma opção para os agricultores do norte do Paraná. 


\section{Referências}

AMAYA-FARFAN, J.; MARCÍLIO, R.; SPEHAR, C. R. Deveria o Brasil investir em novos grãos para a sua alimentação? A proposta do amaranto (Amaranthus sp.) Segurança Alimentar e Nutricional, Campinas, v. 12, n. 1, p. 47-56, jul./dez. 2005.

APAZA-GUTIERREZ, V.; ROMERO-SARAVIA, A.; GUILLÉN-PORTAL, F. R.; BALTERSPERGER, D. D. Response of Grain Amaranth Production to Density and Fertilization in Tarija, Bolivia. In: JANICK, J.; WHIPKEY, A. (Ed.) Trends in new crops and new uses. ASHS Press: Alexandria, 2002. p. 107-109.

AYOADE, J. O. Introdução à climatologia para os trópicos. 9.ed. Rio de Janeiro: Bertrand Brasil, 2003.

BRENNER, D.; WILLIAMS, J. T. Grain amaranth (amaranthus species). In: WILLIAMS, J. T. (Ed.). Underutilized Crops: cereals and pseudocereals. London: Chapman \& Hall, 1995. p. 128-186.

CHAKHATRAKAN, S. Influences of $\mathrm{N}$ fertilizers on the vegetable amaranth production. Thammasat International Journal of Science and Technology, Pathum Thani, v. 8, n. 4, Oct./Dec. 2003. Disponível em: $<$ http://tijsat.tu.ae. th/issues/2003/no4/2003_v8_n04_1.PDF>. Acesso em: 30 jan. 2008.

COSTA, D. M. A.; BORGES, A. S. Avaliação da produção agrícola do amaranto (Amaranthus hypochondriacus). Holos, Natal, v. 21, n. 1, p. 97-111, maio 2005. Disponível em: $\quad<$ http://www.cefetrn.br/ojs/index.php/HOLOS/ article/viewFile/61/67>. Acesso em: 30 jan. 2008.

DOMINGOS, V. D.; ERASMO, E. A. L.; SILVA, J. I. C.; CAVALCANTE, G. D.; SPEHAR, C. R. Crescimento, produção de grãos e biomassa de cultivares de amaranto (Amaranthus cruentus) em função de adubação com NPK. Bioscience Journal, Uberlândia, v. 21, n. 3, p. 2939, sep./dec. 2005.

ERASMO, E. A. L.; DOMINGOS, V. D.; SPEHAR, C. R.; DIDONET, J.; SARMENTO, R. A.; CUNHA, A. M. Avaliação de cultivares de amaranto (Amaranthus spp.) em sistema plantio direto no sul de Tocantins. Bioscience Journal, Uberlândia, v. 20, n. 1, p. 171-176, jan./apr. 2004.

GOMES, F. P. Curso de estatística experimental. Piracicaba: Ed. do autor, 1985.

GUADARRAMA, R. O.; SALGADO, M. T.; SEDEÑO, Y. B. Producción de amaranto con gallinaza en Temoac, Morelos, México. In: CONGRESO NACIONAL DE AGRICULTURA CONSERVACIONISTA, 1., 2002, Temoac. Anais... San José: Universidad Autónoma del Estado de Morelos, 2002. p. 28-29.
HENDERSON, T. L.; JOHNSON, B. L.; SCHNEITER, A. A. Row spacing, plant population and cultivar effects on grain amaranth in the Northern Great Plains. Agronomy Journal, Madison, v. 92, n. 2, p. 329-336, Mar./Apr. 2000.

JOLY, A.B. Botânica: introdução à taxonomia vegetal. 13 ${ }^{\mathrm{a}}$ ed. São Paulo: Companhia Editora Nacional, 2002.

KAUFFMAN, C. S.; WEBER, L. E. Grain amaranth. In: JANICK, J.; SIMON, J. E. (Ed.). Advances in new crops. Portland: Timber Press, 1990. p. 127-139.

MYERS, R. L. Nitrogen fertilizer effect on grain amaranth. Agronomy Journal, Madison, v. 90, n. 5, p. 597-602, Sep./Oct. 1998.

. Amaranth: New crop opportunity. In: JANICK, J. (Ed.). Progress in new crops. Alexandria: ASHS Press, 1996.

SCHULZ-SCHAEFFER, J.; STALLKNECHT, G. F.; BALDRIDGE, D. E. D.; LARSON, R. A. Registration of montana three grain amaranth germplasm. Crop Science, Madison, v. 29, n. 1, p. 244-245, Jan. 1989.

SPEHAR, C. R.; CABEZAS, W. A. R. L. Introdução e seleção de espécies para a diversificação do sistema produtivo nos cerrados. In: CABEZAS, W. A. R. L.; FREITAS, P. L. (Ed.). Plantio direto na integração lavoura pecuária. Uberlândia: Ed. da Universidade Federal de Uberlândia. 2001. p. 179-189.

SPEHAR, C. R.; TEIXEIRA, D. L. Diferenças entre o pseudocereal amaranto e espécies de planta daninha, Amaranthaceae. Planaltina: EMBRAPA Cerrados, 2002. (Comunicado Técnico, 69).

SPEHAR, C. R.; TEIXEIRA, D. L.; CABEZAS, W. A. R. L.; ERASMO, E. A. L. Amaranto BRS Alegria: alternativa para diversificar os sistemas de produção. Pesquisa Agropecuária Brasileira, Brasília, v. 38, n. 5, p. 659-663, maio 2003.

SPEHAR, C. R.; SANTOS, R. L. B.; SOUZA, P. I. M. Novas plantas de cobertura para o sistema de produção de grãos. In: SEMINÁRIO INTERNACIONAL SOBRE O SISTEMA DE PLANTIO DIRETO, 2., 1997, Passo Fundo. Anais... Passo Fundo: Embrapa/CNPT, 1997. p. 169-172, 1997.

TAIZ, L.; ZEIGER, E. Fisiologia vegetal. 3.ed. Porto Alegre: Artmed, 2004.

TEIXEIRA, D. L.; SPEHAR, C. R.; SOUZA, L. A. C. Caracterização agronômica de amaranto para cultivo na entressafra no Cerrado. Pesquisa Agropecuária Brasileira, Brasília, v. 38, n. 1, p. 45-51, jan. 2003. 
